\title{
Prediction of unsaturated shear strength from microstructurally based effective stress
}

\author{
Régis Mpawenayo ${ }^{1,2}$ and Pierre Gerard ${ }^{1, *}$ \\ ${ }^{1}$ Université Libre de Bruxelles (ULB), BATir Department, Brussels, Belgium \\ ${ }^{2}$ Ecole Normale Supérieure, Bujumbura, Burundi
}

\begin{abstract}
This work aims at investigating the adequacy of microstructurally based effective stress to predict the shear strength of unsaturated soils over a wide range of suction. For that purpose, shear strength data are acquired on a silty clay soil through two types of unsaturated triaxial tests: suction controlled triaxial tests and unconsolidated triaxial tests at constant water content. The microstructure of the soil is determined with Mercury Intrusion Porosimetry and is directly used in different expressions of microstructurally based effective stresses available in the literature. The large range of suction tested allows to determine the most consistent expression of the effective stress to reproduce the experimental observations.
\end{abstract}

\section{Introduction}

Shear strength of unsaturated soils can be generally predicted through the definition of an appropriate generalised effective stress [1,2]. It is now well admitted that the generalised effective stress depends on the suction within the sample weighted by an effective stress parameter. This effective stress parameter represents somehow the proportion of the pores where the capillary effects act on the solid grains, and can be thus considered equal to the degree of saturation [3-6] or a function of the degree of saturation $[7,8]$.

However, in remoulded fine-grained soils the compaction process leads to the formation of macropores where capillary effects predominate and micropores where water is attached to the solid through physicochemical bonds $[9,10]$. Consequently, only the free water partially filling the macroporosity plays probably a role on the generalised effective stress, and in turn on the mechanical behaviour of the materials [8]. Those observations have led to the definition of microstructurally based effective stresses where the suction is weighted by an effective degree of saturation characterizing the prevailing suction that contributes to the effective stress [11-13]. On the other hand, in an initially dried clay sample, the micropores saturate first and, then the macropores. That leads to define effective degree of saturation that evolves significantly with high degree of saturation corresponding to the saturation of the macropores once the micropores are fully saturated. A literature review of the strategies used to define microstructurally based effective stress is presented in section 2.

The ability of such microstructurally-based effective stress definitions to predict the mechanical behaviour of different soils has been already investigated in [13]. For that purpose, Mercury Intrusion Porosimetry (MIP) have been achieved to characterize the ratio between the volume of micropores and the total volume of pores, and in turn the degree of saturation of the macropores. They concluded that the stiffness of unsaturated soils can be efficiently predicted from a generalised effective stress expressed in terms of this degree of saturation of the macropores.

Some efforts have been also achieved to predict the unsaturated shear strength from microstructurally based effective stress. Alonso et al [8] proposed a first analysis that illustrates the possible capacity of the methodology. However some elements are not fully considered in this study to provide an integrated analysis of the ability of microstructurally based effective stress: (i) No MIP is performed in this study and the microstructural state variable is thus only a parameter used to optimize the fitting of the shear strength data without any experimental validation; (ii) The range of suctions investigated is limited (i.e. only shear strength data for suctions lower than $600 \mathrm{kPa}$ are used to assess the methodology); (iii) Shear strength data come from direct shear tests and not from triaxial tests.

Consequently, this work aims at investigating the adequacy of microstructurally based effective stress to predict the shear strength of unsaturated soils over a wide range of suctions. To this end shear strength data are acquired on a silty clay soil through two types of unsaturated triaxial tests: suction controlled triaxial tests (using the axis translation technique) and unconsolidated triaxial tests at constant water content for the highest suction levels. The microstructure of the soil is determined by means of MIP and is directly used in different expressions of the effective stress available in the literature. The large range of suctions tested allows to

\footnotetext{
* Corresponding author: piergera@ulb.ac.be
} 
determine the most consistent expression of the effective stress to reproduce the experimental observations.

\section{Expressions of microstructurally based effective stress}

Different expressions of microstructurally based effective stress have been already proposed in the literature. They generally consist in an extension of the Bishop generalised effective stress $\boldsymbol{\sigma}$ ':

$$
\boldsymbol{\sigma}^{\prime}=\boldsymbol{\sigma}+\left(\chi s-u_{a}\right) \boldsymbol{I}
$$

where $\boldsymbol{\sigma}$ is the total stress tensor, $\mathrm{s}$ is the suction, $\chi$ is the effective stress parameter and $\boldsymbol{I}$ is the unity tensor.

The effective stress parameter should represent somehow the proportion of the pores where the capillary effects act on the solid grains. It was often proposed that the degree of saturation $S_{r}$ can be used as a possible candidate for $\chi[1-5 ; 14]$. However, this definition of the effective stress parameter disregards the microstructure of clayey soils, for which two groups of pore sizes are identified: the macropores with predominant capillary effects and micropores with predominant physic-chemical effects. The different mechanisms of interactions between water and the solid phase in the two groups of pores lead to different contributing effect on the constitutive stress.

As a result, some authors proposed to assume that the effective stress parameter $\chi$ is equal to an effective degree of saturation $S_{r, \text { eff }}$ representing the degree of saturation that contributes to the stress measure controlling the mechanical behaviour of clayey soils. The Bishop generalised effective stress becomes thus:

$$
\boldsymbol{\sigma}^{\prime}=\boldsymbol{\sigma}+\left(S_{r, e f f} s-u_{a}\right) \boldsymbol{I}
$$

The determination of $S_{r, \text { eff }}$ requires thus the knowledge of information on the microstructure of the soils, and the progressive saturation of micropores and macropores respectively. Water entering in an initially dried compacted clayey soil will first saturate the micropores because of the strong affinity of the clay platelets with water. However, the saturation of the micropores controlled by physic-chemical effects does not influence the capillary pressure, and so the constitutive stress. Once the micropores are fully saturated, water will saturate progressively the macropores, with effect on the capillary pressure and so on the constitutive stress. Based on that, some authors [8;11-13] proposed to define the effective degree of saturation by a bilinear expression:

$$
\begin{gathered}
S_{r, \text { eff }}=\frac{S_{r}-\zeta_{m}}{1-\zeta_{m}} \text { for } S_{r}>\zeta_{m} \\
S_{r, \text { eff }}=0 \text { for } S_{r} \leq \zeta_{m}
\end{gathered}
$$

where $\zeta_{m}$, called the microstructural state variable, represents the ratio between the porosity of the micropores $n_{m}$ and the total porosity $n$ :

$$
\zeta_{m}=\frac{n_{m}}{n}
$$

A second microstructurally based effective stress proposed in the literature [13] consists in the smoothing of the previous relationship at the transition between the micropores and macropores, which can improve the efficiency of some numerical simulations. It leads to:

$$
S_{r, \text { eff }}=\frac{S_{r}-\zeta_{m}}{1-\zeta_{m}}+\frac{1}{\tau} \ln \left[1+\exp \left(-\tau \frac{S_{r}-\zeta_{m}}{1-\zeta_{m}}\right)\right]
$$

where $\tau$ is a parameter representing the degree of smoothing. This definition assumes that some changes in the effective stress are possible for degree of saturation $S_{r}$ lower than the state variable $\zeta_{m}$. That means that either some macropores start to saturate before the full saturation of the micropores, or the saturation of the micropores leads to some limited changes in the constitutive stress and in turn mechanical behaviour of the soils.

Finally, a third relationship is a power law of the degree of saturation proposed in [8]:

$$
S_{r, e f f}=S_{r}^{\alpha}
$$

Here also the smoothed behaviour is beneficial for numerical calculations, but the loss of direct reference to measured microstructural data of the soils can disregard the principle of microstructurally based effective stress.

Figure 1 presents a comparison between the different relationships.

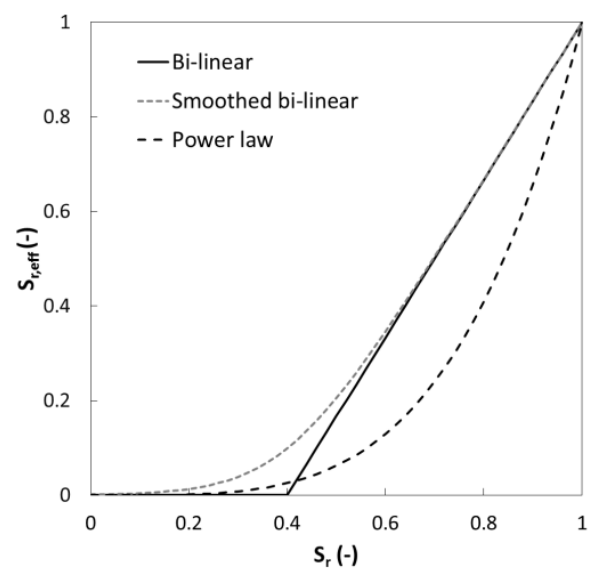

Fig. 1. Comparison of existing microstructurally based effective stresses for $\zeta_{m}=0.4, \tau=7$ and $\alpha=4$.

\section{Test procedure and program}

\subsection{Material}

The soil used in this study is a silty clay composed by $35 \%$ of clay, $27 \%$ of silt and $36 \%$ of sand. The optimum water content and dry density provided by the standard Proctor compaction test are respectively $1.8{\mathrm{~g} . \mathrm{cm}^{-3}}^{-3}$ and $15.6 \%$. The density of solid grains is equal to $2.70 \mathrm{~g} / \mathrm{cm}^{3}$.

\subsection{Preparation of the sample}

All the samples used in this study were dynamically compacted at a dry density of $1,8 \mathrm{~g} \cdot \mathrm{cm}^{-3}$ and an initial 
water content of $12 \%$. The compaction at low initial water content provides generally structured samples with dual porosity [10]. The initial total porosity $n$ is therefore equal to 0.31 .

\subsection{Mercury Intrusion Porosimetry (MIP)}

An initially saturated silty clay soil sample is first dried through freeze-drying technique to do not alter the microstructure [15]. A MIP test in which mercury was first intruded until reaching a maximum pressure of 227 $\mathrm{MPa}$ was conducted on the sample. The pore size distribution of the material can be deduced from the relation between the injection pressure and the volume of mercury injected into the pores.

\subsection{Soil water retention curve}

Two techniques are used to determine the soil water retention curve of the silty clay: the osmotic technique [16] and the chilled-mirror dew-point psychrometer [17].

The osmotic method is a technique of imposition of the suction. Samples with $35 \mathrm{~mm}$ in diameter and $8 \mathrm{~mm}$ high are protected by a semi-permeable membrane and then soaked in a solution of polyethylene glycol 20000 (PEG20000) at a given concentration. Only the movement of water molecules is possible through the membrane and samples reach progressively the imposed suction. Samples are regularly weighted and equalization is assumed when the sample mass stabilizes. The soil volume is then determined by means of a $3 \mathrm{D}$ scanning tool. This technique is used for suction ranging from 0 to $2 \mathrm{MPa}$.

The chilled-mirror dew-point psychrometer is a technique for measuring suction. Samples of $35 \mathrm{~mm}$ in diameter and $8 \mathrm{~mm}$ in height are air dried until different water contents. Then the measurement of the suction was done using a WP4C water potential meter. The use of the chilled mirror method allows determining the relative humidity of the air around the sample installed in a sealed chamber once the sample has come into equilibrium with the vapour in the surrounding air. The dew-point psychrometer is used for determination of suction above $2 \mathrm{MPa}$.

\subsection{Triaxial test}

Samples, $36 \mathrm{~mm}$ in diameter and $70 \mathrm{~mm}$ high, were dynamically compacted in a mould in 3 layers of same thickness for the different triaxial tests presented hereafter.

\subsubsection{Consolidated and drained (CD) saturated triaxial test}

Conventional $\mathrm{CD}$ triaxial test are achieved under saturated conditions. The samples are first saturated using confining pressure and water pressure at the top of the sample of respectively $60 \mathrm{kPa}$ and $55 \mathrm{kPa}$. The saturation of the samples is assumed when the Skempton coefficient $B$ reaches a value of 0.95 . After the consolidation at different normal pressures the samples are sheared with the same confinement as during the consolidation and at a constant strain rate of $0.007 \mathrm{~mm} / \mathrm{min}$ (NF P94-074). The cross-sectional area under loading was calculated according to Head [18].

\subsubsection{Suction-controlled triaxial test}

The axis translation technique is used to impose constant suction within the samples during shearing [19]. To this end the conventional triaxial cell has been modified. A high-air-entry ceramic disk (HAED) was sealed onto the base pedestal of the cell. It allows a separate control for the air pressure and the water pressure when the HAED is fully saturated (i.e. only water can pass through it). In our study HAED with air entry pressure of $500 \mathrm{kPa}$ has been used. However, dissolved air can pass through HAED and air can form below HAED and progressively desaturate the porous disk. A flushing system is added to remove the air bubbles beneath the porous stone. A spiral drainage groove of $2 \mathrm{~mm}$ of diameter and $0.5 \mathrm{~mm}$ of height is included on the bottom face of the porous disk to ensure an efficient trapping of the air.

The samples are first saturated using the same procedure as for the CD triaxial tests. After consolidation, different suctions are imposed through the axis translation technique. Homogeneous suction within the sample is assumed when the volume of water expelled from the sample is lower to $0.1 \mathrm{~cm}^{3}$ per day [20]. Finally shearing of the samples is achieved in drained conditions with a vertical strain rate of $0.025 \mathrm{~mm} / \mathrm{h}$ [21]. The evolution of the section is determined assuming the change in volume of the confining fluid corresponds to the change in volume of the sample.

For each imposed suctions, 3 different confining pressures are imposed and tested.

\subsubsection{Constant water content triaxial test}

Constant water content triaxial tests are achieved to analyse the shear strength for suctions higher than 500 $\mathrm{kPa}$. After the same procedure of samples saturation as described above, the samples are dried in a desiccator where evaporation is controlled by means of a saline solution [22]. Such a drying procedure is preferred to the air-drying to limit the gradient of moisture within the specimen and in turn the specimen cracking. The samples are removed from the desiccator when a target mass is reached. It means that the samples are not necessary in equilibrium with the relative humidity imposed when they are removed. The specimen is then covered with a plastic film and an aluminium foil and are wrapped with a paraffin layer for one week to homogenize the water content and prevent evaporation during this step. Finally, the samples are sheared in a triaxial cell under undrained conditions at a constant strain rate of $0.5 \mathrm{~mm} / \mathrm{min}$. This high strain rate allows assuming that specimen remain at a constant water content during shearing.

Since the impact of confining pressure on shear strength becomes negligible for high suctions, only one 
confining pressure is tested for each constant water content/suction.

\section{Experimental results}

\subsection{Pore size distribution}

Figure 2 presents the pore size distribution of the "ascompacted" silty clay soil. A bimodal distribution can be distinguished. From this figure, the diameter of critical pores marking the transition between micropores and macropores can be determined, and is considered to be equal to $0.15 \mathrm{~mm}$. From the cumulative intrusion curve, it is then possible to calculate the porosity of the macropores $\mathrm{n}_{\mathrm{M}}$ equal to 0.15 .

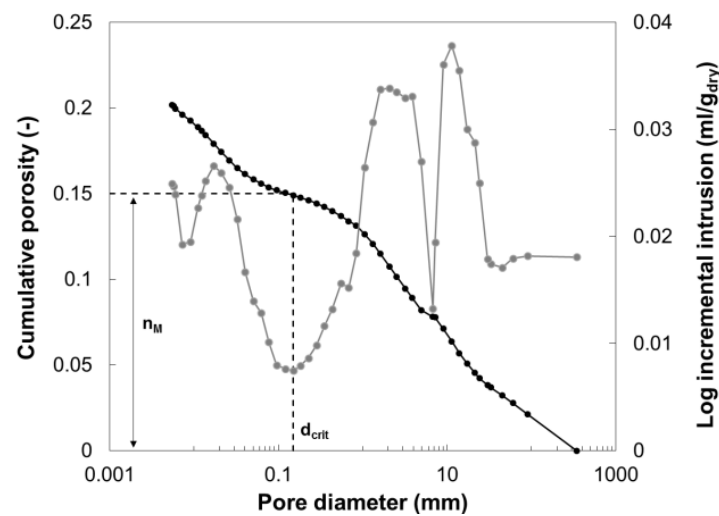

Fig. 2. MIP result on as-compacted silty clay

\subsection{Soil water retention curve}

Figure 3 presents the soil water retention curve of the silty clay soil obtained from osmotic technique and chilledmirror dew-point psychrometer.

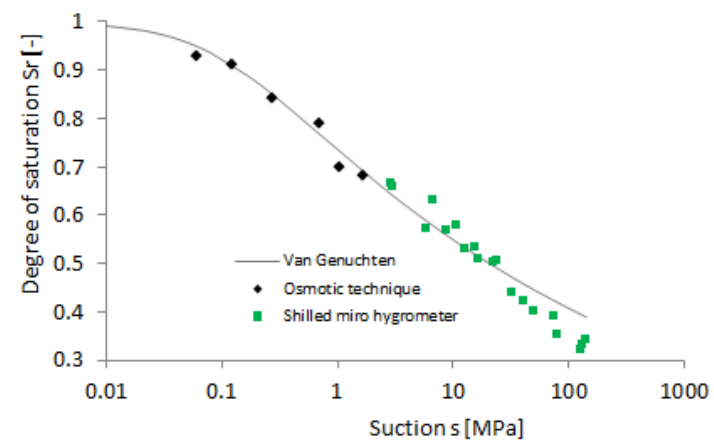

Fig. 3. Soil water retention curve of the silty soil

A van Genuchten retention model is fitted on the experimental data. It leads to

$$
S_{r}=\left[1+\left(\frac{S}{P_{r}}\right)^{\lambda}\right]^{-\left(1-\frac{1}{\lambda}\right)}
$$

with $\lambda=1.13$ and $\mathrm{P}_{\mathrm{r}}=100 \mathrm{kPa}$.

\subsection{Saturated shear strength}

$3 \mathrm{CD}$ triaxial tests under saturated conditions have been achieved at 3 different confining pressures $(60,100$ and $200 \mathrm{kPa}$ ). The shear failure is considered occurring at the maximum deviatoric stress $q$. This value allows to determine the mean effective stress $p^{\prime}\left(=q / 3+\sigma_{3}^{\prime}\right)$ at failure for each confining pressure (Figure 4$)$. It leads to a shear strength criterion:

$$
q=m p+k
$$

with $m=0.80$ and $k=19.2$, corresponding to an effective cohesion $c^{\prime}$ and an effective friction angle $\varphi^{\prime}$ of respectively $9 \mathrm{kPa}$ and $21^{\circ}$.

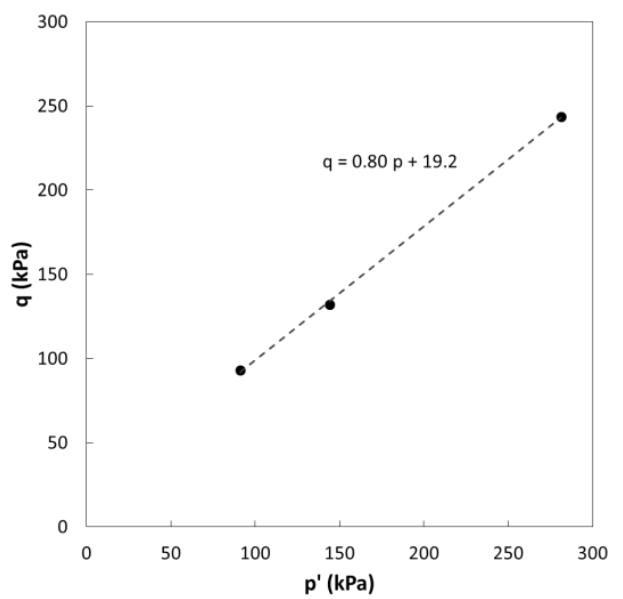

Fig. 4. Shear failure criterion under saturated conditions.

Table 1. Unsaturated shear strength. ( $\mathrm{SC}=$ suction-controlled triaxial test; $\mathrm{CWC}=$ constant water content triaxial test)

\begin{tabular}{|c|c|c|c|c|}
\hline Test & $\mathbf{s}(\mathbf{k P a})$ & $\boldsymbol{\sigma}_{3}(\mathbf{k P a})$ & $\mathbf{q}(\mathbf{k P a})$ & $\mathbf{S}_{\mathbf{r}}(\mathbf{-})$ \\
\hline SC & 30 & 60 & 90 & 0.97 \\
\hline SC & 30 & 90 & 124 & 0.97 \\
\hline SC & 30 & 130 & 167 & 0.97 \\
\hline SC & 100 & 130 & 144 & 0.93 \\
\hline SC & 100 & 160 & 183 & 0.93 \\
\hline SC & 100 & 200 & 224 & 0.93 \\
\hline SC & 250 & 280 & 220 & 0.85 \\
\hline SC & 250 & 310 & 230 & 0.85 \\
\hline SC & 250 & 350 & 280 & 0.85 \\
\hline CWC & 1650 & 150 & 674 & 0.69 \\
\hline CWC & 2920 & 150 & 799 & 0.64 \\
\hline CWC & 4950 & 150 & 1001 & 0.60 \\
\hline CWC & 11300 & 150 & 1200 & 0.54 \\
\hline CWC & 22500 & 150 & 1272 & 0.49 \\
\hline CWC & 45300 & 150 & 1787 & 0.45 \\
\hline
\end{tabular}

\subsection{Unsaturated shear strength}

Unsaturated shear strength has been determined for various suctions from suction-controlled and constant water content triaxial tests. Table 1 summarize the unsaturated shear strength obtained from the different tests. For suction-controlled triaxial test, the degree of 
saturation $\mathrm{S}_{\mathrm{r}}$ is calculated from the van Genuchten retention curve using the imposed suction. For constant water content triaxial test, a sample is extracted from the specimen at the end of the test, and the water content is determined from weighting. The suction is then calculated from the van Genuchten model of the soil-water retention curve (eq. 7)

\section{Discussion}

Using the MIP results and eq. 4, a microstructural state variable $\zeta_{m}$ equal to 0.52 is obtained. It is then possible to determine the mean effective stress $p$ ' at failure for the different unsaturated triaxial tests, using the different definitions of microstructurally based effective stresses presented in section 2. It becomes:

$$
p^{\prime}=\sigma_{3}+\frac{q}{3}-u_{a}+S_{r, e f f} s
$$

with $\mathrm{S}_{\mathrm{r} \text {,eff }}$ obtained from equations 3, 5 or 6 respectively.

With an appropriate definition of the effective stress for unsaturated conditions, a unique shear failure criterion should be able to predict the effective stress state at failure both for saturated and unsaturated conditions. In this study, the shear failure criterion under saturated conditions has been determined in section 4.3 through saturated CD triaxial tests. It is thus proposed to identify the most suitable expression of microstructurally based effective stress through a comparison between the effective stress state at failure measured in the different unsaturated triaxial tests (eq. 9) and the prediction coming from the saturated shear strength criterion.

Figures 5 to 7 present the comparison between the experimental effective stress state at failure for the unsaturated triaxial tests and the prediction of the stress at failure using the microstructurally based effective stress definitions and the saturated shear strength. The bilinear expression (eq. 3) is used in Figure 5. On Figure 6 a smoothed expression of the bilinear equation (eq. 5) is considered, with the smoothing coefficient $\tau$ fitted on experimental data and equal to 8. Finally, a power law expression (eq. 6) is envisaged, with a coefficient $\alpha$ equal to 4.13 .

The results highlight first that the three models seem suitable to predict the unsaturated shear strength for low values of the suctions $(<200 \mathrm{kPa})$. Then it demonstrates the inability of the bilinear equation to predict the unsaturated shear strength for large suctions (and so large p'). Also the smoothed bilinear relation and the power law expression seem to be good candidates for a suitable expression of a microstructurally-based effective stress for the full range of suctions tested in this study.

Finally, for each unsaturated triaxial test, the "back calculated effective stress parameter" necessary to fulfil the uniqueness of the effective-stress dependent shear failure criterion under both saturated and unsaturated conditions is calculated:

$$
S_{r, \text { eff }}=\frac{\frac{q_{\text {exp }, \text { failure }}-k}{m}-p_{\text {failure }}+u_{a}}{s}
$$

with $q_{\text {exp,failure }}$ and $p_{\text {failure }}$ the experimental deviatoric stress and the total mean stress at failure determined in the unsaturated triaxial tests.

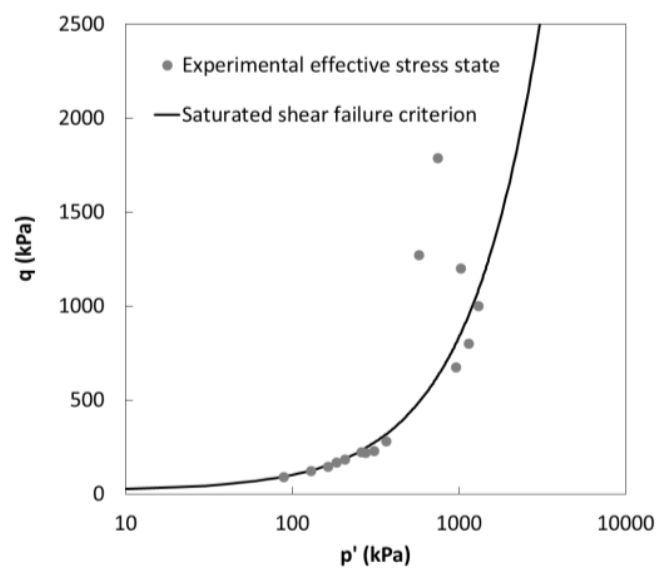

Fig. 5. Comparison between experimental effectives stress determined from microstructurally based effective stress (bilinear relation - eq. 3 ) and prediction from saturated shear failure criterion

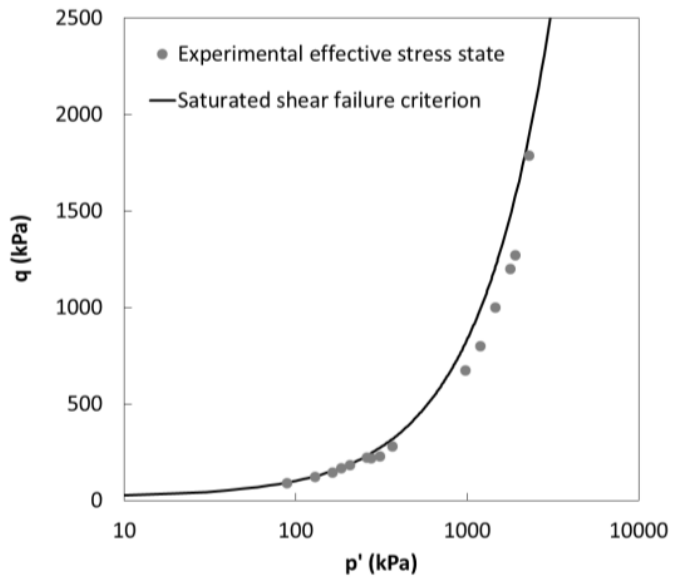

Fig. 6. Comparison between experimental effectives stress determined from microstructurally based effective stress (smoothed bilinear relation - eq. 5) and prediction from saturated shear failure criterion $-\tau=8$

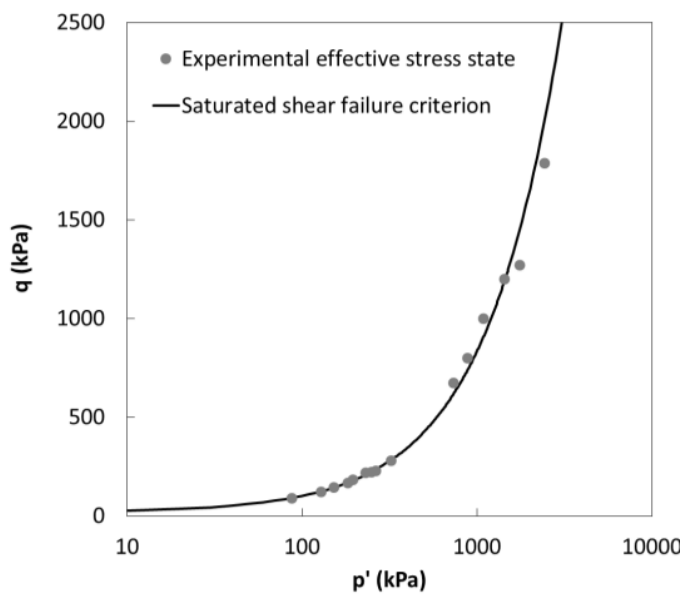

Fig. 7. Comparison between experimental effectives stress determined from microstructurally based effective stress (power law relation - eq. 6) and prediction from saturated shear failure criterion $-\alpha=4.13$ 
Figure 8 presents the experimental unsaturated shear strength data expressed in terms of $S_{r, \text { eff }}-S_{r}$, and their comparison with the 3 microstructurally based effective stress models presented in section 2 (eq. 3, 5 and 6). This figure provides a complementary information to Figures 5 to 7. It illustrates for example that the power law expression better fits the unsaturated shear strength than the smoothed bilinear model for small suctions (i.e. $S_{r}>$ $0.8)$.

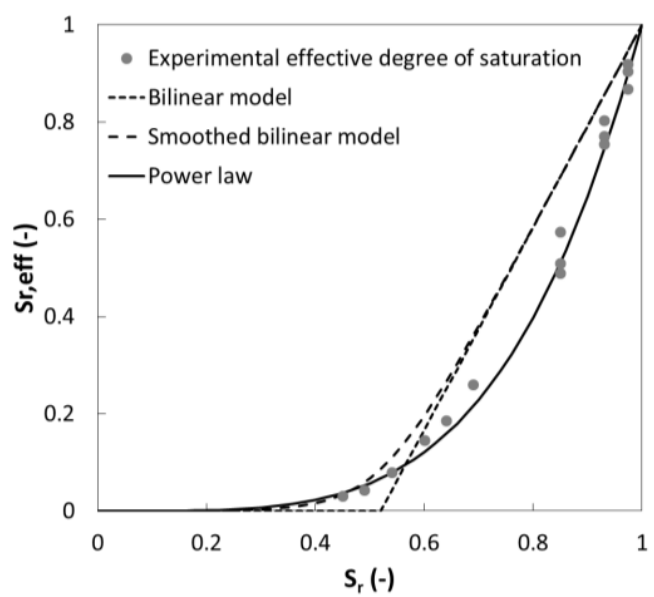

Fig. 8. Comparison between experimental effective degree of saturation (to fulfil the uniqueness of the shear failure criterion) and the expressions provided by microstructurally-based effective stress expressions.

\section{Conclusions}

This study aims at investigating the adequacy of microstructurally based effective stress to predict the shear strength of unsaturated soils. The main contribution of this work is to assess the suitability of the microstructurally based expressions over a wide range of suctions. To this end shear strength data are obtained on a silty clay soil through suction-controlled triaxial tests and unconsolidated triaxial tests at constant water content. The microstructure of the soil is determined by means of mercury intrusion porosimetry and is directly used in different expressions of the effective stress available in the literature. The results illustrate the adequacy of a power law expression of the effective stress to predict the unsaturated shear strength. However, even if such an expression is based on a physical explanation on the role of the microstructure on the effective stress, and in turn on the shear strength, there is no direct reference to measured microstructural data in this model. The capacity of prediction of unsaturated shear strength only based on microstructural data is therefore limited.

On the other hand, the bilinear equation uses directly microstructural data of the soil and is more physically based, but fails in reproducing experimental unsaturated shear strength. That can be explained by more complex saturation processes of the microstructure than the one described in the introduction. The saturation of the micropores is probably not only associated with physicchemical processes, and can slightly modify the capillary pressures, and so affects the effective stress, also for small degrees of saturation. Also the bilinear formulation (as the two other microstructurally-based effective stresses) neglects the interfacial forces between air and water. Nikooee et al. [23] and Likos [24] showed that interfacial effects are much more pronounced at high suctions and in fine-grained soils. On Fig.5, the main discrepancies are observed for high suction. Such an effect should be therefore considered in future works to validate the expression of a microstructurally-based effective stress for large suctions.

\section{References}

1. M.A. Bishop, Tek. Ukebl. 106, 39 (1959)

2. M. Nuth, L. Laloui, Int. J. Numer. Anal. Methods Geomech. 24, 11 (2007)

3. C. Jommi, Experimental Evidence and theoretical approaches in unsaturated soils, Proceedings of the international workshop on unsaturated soils, 139-153 (2000)

4. B. Loret, N. Khalili, Int. J. Numer. Anal. Methods Geomech. 24, 11 (2000)

5. J.M. Pereira, H. Wong, P. Dubujet, P. Dangla, Int. J. Numer. Anal. Methods Geomech. 29, 11 (2005)

6. D. Sheng, S. Sloan, A. Gens Comput. Mech. 33, 6 (2004)

7. O. Coussy, J.M. Pereira, J. Vaunat. Comput. Geotech. 37 (2010)

8. E.E. Alonso, J.M. Pereira, J. Vaunat, S. Olivella, Géotechnique. 60, 12 (2010)

9. P. Delage, M. Audiger, Y.J. Cui, M. Howat, Can. Geotech. J. 33, 1 (1996)

10. E. Romero, P. Simms, Geotech. Geol. Engng. 26, 6 (2008)

11. E. Romero, J. Vaunat, Experimental Evidence and theoretical approaches in unsaturated soils, Proceedings of the international workshop on unsaturated soils, 91-106 (2000)

12. A. Tarantino, S. Tombolato, Géotechnique. 58, 3 (2005)

13. E.E. Alonso, N.M. Pinyol, A. Gens, Géotechnique. 63, 6 (2013)

14. J. Jennings, J. Burland, Géotechnique. 12, 2 (1962)

15. M. Oualmakran, B.C.N. Mercatoris, B. François, Can. Geotech. J. 53, 12 (2016)

16. G. Khassif, A. Ben Shalom, Géotechnique. 21 (1971)

17. N. Lu, W.J. Likos, Unsaturated soil mechanics. John Wiley \& Sons (2004)

18. K.H. Head, Manual of soil laboratory testing (1986)

19. S.K. Vanapalli, M.V. Nicotera, R.S. Sharma, Geotech. Geol. Engng. 26, 6 (2008)

20. A.R. Estrabragh, A.A. Javadi, Can. Geotech. J. 45, 3 (2008)

21. P. Delage, Experimental unsaturated soil mechanics, Proceedings of the international conference on unsaturated soils, 973-996 (2002)

22. P. Delage, H.D. Howat, Y.J. Cui, Eng. Geol. 50 (1998)

23. E. nikooee, G. Habibagahi, S.M. Hassanizadeh, A. Ghahramani, Transport Porous Med. 96, 2 (2013).

24. W.J. Likos, Vadose Zone J. 13, 5 (2014) 